\title{
Triple Coincidence Point Theorems for Multi - Valued Maps in Partially Ordered Metric Spaces
}

\author{
K.P.R.Rao ${ }^{1}$, G.N.V.Kishore ${ }^{2, *}$, P.R.Sobhana Babu ${ }^{3}$ \\ ${ }^{1}$ Department of Mathematics, Acharya Nagarjuna University,Nagarjuna Nagar, \\ Guntur - 522 510, Andhra Pradesh, India \\ ${ }^{2}$ Department of Mathematics, Baba Institute of Technology and Sciences,P.M.Palem, \\ Madhurawada Visakhapatnam - 530048, Andhra Pradesh, India \\ ${ }^{3}$ Department of Mathematics, Ramachandra College of Engineering, Vatluru(V), \\ Eluru-534007, West Godavari Dist., Andhra Pradesh, India \\ ${ }^{*}$ Corresponding Author: kishore.apr2@gmail.com
}

Copyright (C2013 Horizon Research Publishing All rights reserved.

\begin{abstract}
In this paper we prove a triple coincidence point theorem for multi - valued and single-valued mappings in a partially ordered metric space based on the concepts of [5]. Also we give an example which supports our main result. Our result generalizes several results relating to coupled fixed point theorems.
\end{abstract}

Keywords Triple fixed point, complete space, $w$ - compatible, set-valued mapping, $\Delta$ - Symmetric Property

2000 Mathematics Subject Classification: 54H25, 47H10, 54E50

\section{Introduction}

The study of fixed points for multi - valued contraction mappings using the Hausdorff metric was initiated by Nadler [9].

Let $(X, d)$ be a metric space. We denote $C B(X)$ the family of all non - empty closed and bounded sub sets of $X$ and $C L(X)$ the set of all non - empty closed sub sets of $X$. For $A, B \in C B(X)$ and $x \in X$, we denote $D(x, A)=\inf \{d(x, a): a \in A\}$. Let $H$ be the Hausdorff metric induced by the metric $d$ on $X$, that is

$$
H(A, B)=\max \left\{\sup _{x \in A} d(x, B), \sup _{y \in B} d(y, A)\right\}
$$

for every $A, B \in C B(X)$.

Definition 1.1 An element $x \in X$ is said to be a fixed point of a set-valued mapping $T: X \rightarrow C B(X)$ if and only if $x \in T x$.

In 1969, Nadler [9] extended the famous Banach Contraction Principle [8] from single - valued mapping to multi - valued mapping and proved the following fixed point theorem for the multi - valued contraction.

Theorem $1.2([9])$ : Let $(X, d)$ be a complete metric space and let $T$ be a mapping from $X$ into $C B(X)$. Assume that there exists $c \in[0,1)$ such that

$$
H(T x, T y) \leq c d(x, y),
$$

for all $x, y \in X$. Then, $T$ has a fixed point.

The existence of fixed points for various multi - valued contractive mappings has been studied by many authors under different conditions. For details, we refer the reader to $[1,4,7,9,11]$ and the references therein.

The concept of coupled fixed point for multi - valued mapping was introduced by Samet and Vetro [2] and later several authors namely Hussain and Alotaibi [6] and Aydi et. al.[3] proved coupled coincidence point theorems in partially ordered metric spaces.

Berinde and Borcut [10], introduced the concept of triple fixed points and obtained a tripled fixed point theorem for single valued map. Later we introduced Triple fixed, Triple coincidence and Triple common fixed points for multi - valued maps in our earlier paper [5] as follows. 
Definition 1.3 ([5]) Let $X$ be a non empty set, $T: X \times X \times X \rightarrow 2^{X}$ (Collection of all non empty subsets of $X$ ). $f: X \rightarrow X$.

(i) The point $(x, y, z) \in X \times X \times X$ is called a tripled fixed of $T$ if

$$
x \in T(x, y, z), y \in T(y, x, y) \text { and } z \in T(z, y, x) .
$$

(ii) The point $(x, y, z) \in X \times X \times X$ is called a tripled coincident point of $T$ and $f$ if

$$
f x \in T(x, y, z), f y \in T(y, x, y) \text { and } f z \in T(z, y, x) .
$$

(iii) The point $(x, y, z) \in X \times X \times X$ is called a tripled common fixed point of $T$ and $f$ if

$$
x=f x \in T(x, y, z), y=f y \in T(y, x, y) \text { and } z=f z \in T(z, y, x) .
$$

Definition 1.4 ([5]) Let $T: X \times X \times X \rightarrow 2^{X}$ be a multi - valued map and $f$ be a self map on $X$. The Hybrid pair $\{T, f\}$ is called $w$ - compatible if $f(T(x, y, z)) \subseteq T(f x, f y, f z)$ whenever $(x, y, z)$ is a tripled coincidence point of $T$ and $f$.

\section{Results}

Let $(X, d)$ be a metric space endowed with a partial order $\preceq$ and $G: X \rightarrow X$. Define the set $\Delta \subset X^{3}$ by $\Delta=\left\{(x, y, z) \in X^{3}:\right.$ Gx R Gy, Gx R Gz and Gy R Gz .

Definition 2.1 A mapping $F: X^{3} \rightarrow X$ is said to have a $\Delta$ - Symmetric property if and only if $(x, y, z) \in \Delta \Rightarrow$ $F(x, y, z) R F(y, x, y), F(x, y, z) R F(z, y, x)$ and $F(y, x, y) R F(z, y, z)$.

Definition 2.2 A mapping $f: X^{3} \rightarrow[0, \infty)$ is called lower semi continuous if, for any sequences $\left\{x_{n}\right\},\left\{y_{n}\right\},\left\{z_{n}\right\}$ in $X$ and $(x, y, z) \in X^{3}$, one has

$$
\lim _{n \rightarrow \infty}\left(x_{n}, y_{n}, z_{n}\right)=(x, y, z) \Longrightarrow f(x, y, z) \leq \lim _{n \rightarrow \infty} \inf f\left(x_{n}, y_{n}, z_{n}\right) .
$$

Theorem 2.3 Let $(X, d)$ be a metric space endowed with a partial order $\preceq$ and $\Delta \neq \emptyset$. Suppose that $F: X \times$ $X \times X \rightarrow C L(X)$ has a $\Delta$ - Symmetric property, $g: X \rightarrow X$ is continuous, $g(X)$ is complete, the function $f: g(X) \times g(X) \times g(X) \rightarrow[0,+\infty)$ defined for all $x, y, z \in X$ by

(2.3.1) $f(g x, g y, g z):=D(g x, F(x, y, z))+D(g y, F(y, x, y))+D(g z, F(z, y, x))$,

is lower semi - continuous and that there exists a function $\phi:[0,+\infty) \rightarrow[a, 1), 0<a<1$, satisfying

(2.3.2) $\lim _{r \rightarrow t^{+}} \sup \phi(r)<1$, for each $t \in[0,+\infty)$.

Assume that for any $(x, y, z) \in \Delta$ there exist $g u \in F(x, y, z), g v \in F(y, x, y)$ and $g w \in F(z, y, x)$ satisfying

(2.3.3) $\sqrt{\phi(f(g x, g y, g z))}[d(g x, g u)+d(g y, g v)+d(g z, g w)] \leq f(g x, g y, g z)$ such that

(2.3.4) $f(g u, g v, g w) \leq \phi(f(g x, g y, g z))[d(g x, g u)+d(g y, g v)+d(g z, g w)]$.

Then, $F$ and $g$ have a triple coincidence point. That is, there exists $(g \alpha, g \beta, g \gamma) \in X \times X \times X$ such that $g \alpha \in F(\alpha, \beta, \gamma), g \beta \in F(\beta, \alpha, \beta)$ and $g \gamma \in F(\gamma, \beta, \alpha)$.

Let $\left(x_{0}, y_{0}, z_{0}\right) \in \Delta$ be arbitrary and fixed, by (2.3.3) and (2.3.4), we can choose $g x_{1} \in F\left(x_{0}, y_{0}, z_{0}\right), g y_{1} \in$ $F\left(y_{0}, x_{0}, y_{0}\right)$ and $g z_{1} \in F\left(z_{0}, y_{0}, x_{0}\right)$ such that

$$
\sqrt{\phi\left(f\left(g x_{0}, g y_{0}, g z_{0}\right)\right)}\left[d\left(g x_{0}, g x_{1}\right)+d\left(g y_{0}, g y_{1}\right)+d\left(g z_{0}, g z_{1}\right)\right] \leq f\left(g x_{0}, g y_{0}, g z_{0}\right)
$$

and

$$
f\left(g x_{1}, g y_{1}, g z_{1}\right) \leq \phi\left(f\left(g x_{0}, g y_{0}, g z_{0}\right)\right)\left[d\left(g x_{0}, g x_{1}\right)+d\left(g y_{0}, g y_{1}\right)+d\left(g z_{0}, g z_{1}\right)\right] .
$$

From (2.1) and (2.2), we get

$$
\begin{aligned}
f\left(g x_{1}, g y_{1}, g z_{1}\right) & \leq \phi\left(f\left(g x_{0}, g y_{0}, g z_{0}\right)\right)\left[d\left(g x_{0}, g x_{1}\right)+d\left(g y_{0}, g y_{1}\right)+d\left(g z_{0}, g z_{1}\right)\right] \\
& =\sqrt{\phi\left(f\left(g x_{0}, g y_{0}, g z_{0}\right)\right)}\left\{\sqrt{\phi\left(f\left(g x_{0}, g y_{0}, g z_{0}\right)\right)}\right. \\
& \leq \sqrt{\left.\left.\phi\left(g x_{0}, g x_{1}\right)+d\left(g y_{0}, g y_{1}\right)+d\left(g z_{0}, g z_{1}\right)\right]\right\}} \\
& =\sqrt{\left.\phi\left(g x_{0}, g z_{0}\right)\right)} f\left(g x_{0}, g y_{0}, g z_{0}\right) .
\end{aligned}
$$


Thus

$$
f\left(g x_{1}, g y_{1}, g z_{1}\right) \leq \sqrt{\phi\left(f\left(g x_{0}, g y_{0}, g z_{0}\right)\right)} f\left(g x_{0}, g y_{0}, g z_{0}\right) .
$$

Now, since $F$ has a $\Delta$ - Symmetric property and $\left(x_{0}, y_{0}, z_{0}\right) \in \Delta$, we have

$$
F\left(x_{0}, y_{0}, z_{0}\right) R F\left(y_{0}, x_{0}, y_{0}\right), F\left(x_{0}, y_{0}, z_{0}\right) R F\left(z_{0}, y_{0}, x_{0}\right) \text { and } F\left(y_{0}, x_{0}, y_{0}\right) R F\left(z_{0}, y_{0}, x_{0}\right)
$$

Thus

$$
g x_{1} R g y_{1}, g x_{1} R g z_{1} \text { and } g y_{1} R g z_{1}
$$

By definition of $\Delta,\left(x_{1}, y_{1}, z_{1}\right) \in \Delta$.

Again from (2.3.3) and (2.3.4), we can choose $g x_{2} \in F\left(x_{1}, y_{1}, z_{1}\right), g y_{2} \in F\left(y_{1}, x_{1}, y_{1}\right)$ and $g z_{2} \in F\left(z_{1}, y_{1}, x_{1}\right)$ such that

$$
\sqrt{\phi\left(f\left(g x_{1}, g y_{1}, g z_{1}\right)\right)}\left[d\left(g x_{1}, g x_{2}\right)+d\left(g y_{1}, g y_{2}\right)+d\left(g z_{1}, g z_{2}\right)\right] \leq f\left(g x_{1}, g y_{1}, g z_{1}\right)
$$

and

$$
f\left(g x_{2}, g y_{2}, g z_{2}\right) \leq \phi\left(f\left(g x_{1}, g y_{1}, g z_{1}\right)\right)\left[d\left(g x_{1}, g x_{2}\right)+d\left(g y_{1}, g y_{2}\right)+d\left(g z_{1}, g z_{2}\right)\right]
$$

Hence, we get

$$
f\left(g x_{2}, g y_{2}, g z_{2}\right) \leq \sqrt{\phi\left(f\left(g x_{1}, g y_{1}, g z_{1}\right)\right)} f\left(g x_{1}, g y_{1}, g z_{1}\right), \text { with }\left(x_{2}, y_{2}, z_{2}\right) \in \Delta .
$$

Continuing this process we can choose $g x_{n} \in X, g y_{n} \in X$ and $g z_{n} \in X$ such that for all $n=0,1,2, \cdots$, we have $\left(x_{n}, y_{n}, z_{n}\right) \in \Delta$

$$
\begin{gathered}
g x_{n+1} \in F\left(x_{n}, y_{n}, z_{n}\right), g y_{n+1} \in F\left(y_{n}, x_{n}, y_{n}\right) \text { and } g z_{n+1} \in F\left(z_{n}, y_{n}, x_{n}\right), \\
\sqrt{\phi\left(f\left(g x_{n}, g y_{n}, g z_{n}\right)\right)}\left[\begin{array}{c}
d\left(g x_{n}, g x_{n+1}\right) \\
+d\left(g y_{n}, g y_{n+1}\right) \\
+d\left(g z_{n}, g z_{n+1}\right)
\end{array}\right] \leq f\left(g x_{n}, g y_{n}, g z_{n}\right)
\end{gathered}
$$

and

$$
f\left(g x_{n+1}, g y_{n+1}, g z_{n+1}\right) \leq \sqrt{\phi\left(f\left(g x_{n}, g y_{n}, g z_{n}\right)\right)} f\left(g x_{n}, g y_{n}, g z_{n}\right)
$$

with $\left(x_{n+1}, y_{n+1}, z_{n+1}\right) \in \Delta$.

Now, we shall show that $f\left(g x_{n}, g y_{n}, g z_{n}\right) \rightarrow 0$ as $n \rightarrow \infty$.

If $f\left(g x_{m}, g y_{m}, g z_{m}\right)=0$, for some $m$, then we get

$$
\begin{array}{r}
D\left(g x_{m}, F\left(x_{m}, y_{m}, z_{m}\right)\right)=0, \text { implies that } g x_{m} \in \overline{F\left(x_{m}, y_{m}, z_{m}\right)}=F\left(x_{m}, y_{m}, z_{m}\right), \\
D\left(g y_{m}, F\left(y_{m}, x_{m}, y_{m}\right)\right)=0, \text { implies that } g y_{m} \in \overline{F\left(y_{m}, x_{m}, y_{m}\right)}=F\left(y_{m}, x_{m}, y_{m}\right) \\
\text { and } \\
D\left(g z_{m}, F\left(z_{m}, y_{m}, x_{m}\right)\right)=0, \text { implies that } g z_{m} \in \overline{F\left(z_{m}, y_{m}, x_{m}\right)}=F\left(z_{m}, y_{m}, x_{m}\right) .
\end{array}
$$

Hence in this case $\left(g x_{m}, g y_{m}, g z_{m}\right)$ is a triple coincidence point of $F$ and $g$ and the theorem is proved.

Suppose that $f\left(g x_{n}, g y_{n}, g z_{n}\right)>0$ for all $n$.

Using (2.7) and $\phi(t)<1$, we conclude that $\left\{f\left(g x_{n}, g y_{n}, g z_{n}\right)\right\}$ is a strictly decreasing sequence of non - negative real numbers. Thus, there exists a $\delta \geq 0$, such that

$$
\lim _{n \rightarrow \infty} f\left(g x_{n}, g y_{n}, g z_{n}\right)=\delta .
$$

Now, we will show that $\delta=0$. On contrary assume that $\delta>0$,

Letting $n \rightarrow \infty$ in (2.7), we have that

$$
\delta \leq \lim _{f\left(g x_{n}, g y_{n}, g z_{n}\right) \rightarrow \delta^{+}} \sup \sqrt{\phi\left(f\left(g x_{n}, g y_{n}, g z_{n}\right)\right)} \delta<\delta,
$$

a contradiction. Hence $\delta=0$. That is

$$
\lim _{n \rightarrow \infty} f\left(g x_{n}, g y_{n}, g z_{n}\right)=0 .
$$

Now, we prove $\left\{g x_{n}\right\},\left\{g y_{n}\right\}$ and $\left\{g z_{n}\right\}$ are Cauchy sequences in $(X, d)$.

Suppose

$$
\delta \leq \lim _{f\left(g x_{n}, g y_{n}, g z_{n}\right) \rightarrow 0^{+}} \sup \sqrt{\phi\left(f\left(g x_{n}, g y_{n}, g z_{n}\right)\right)} .
$$

Then by assumption (2.3.2), we have $\delta<1$.

Let $k$ be such that $\delta<k<1$. Then, there exists $n_{0} \in N$ such that

$$
\sqrt{\phi\left(f\left(g x_{n}, g y_{n}, g z_{n}\right)\right)}<k, \text { for each } n \geq n_{0}
$$

Thus from (2.7), we get

$f\left(g x_{n+1}, g y_{n+1}, g z_{n+1}\right)<k . f\left(g x_{n}, g y_{n}, g z_{n}\right)$, for each $n \geq n_{0}$. 
Hence by induction, for each $n \geq n_{0}$, we have

$$
f\left(g x_{n+1}, g y_{n+1}, g z_{n+1}\right)<k^{n+1-n_{0}} f\left(g x_{n_{0}}, g y_{n_{0}}, g z_{n_{0}}\right) .
$$

Since $\phi(t) \geq a>0$ for all $t>0$, from (2.6), (2.9) and for each $n \geq n_{0}$, we have

$$
d\left(g x_{n}, g x_{n+1}\right)+d\left(g y_{n}, g y_{n+1}\right)+d\left(g z_{n}, g z_{n+1}\right)<\frac{1}{\sqrt{a}} k^{n-n_{0}} f\left(g x_{n_{0}}, g y_{n_{0}}, g z_{n_{0}}\right) .
$$

Now we consider for $m>n$, we have

$$
\begin{aligned}
& d\left(g x_{n}, g x_{m}\right)+d\left(g y_{n}, g y_{m}\right)+d\left(g z_{n}, g z_{m}\right) \\
& \leq \quad d\left(g x_{n}, g x_{n+1}\right)+d\left(g x_{n+1}, g x_{n+2}\right)+\cdots+d\left(g x_{m-1}, g x_{m}\right) \\
&+d\left(g y_{n}, g y_{n+1}\right)+d\left(g y_{n+1}, g y_{n+2}\right)+\cdots+d\left(g y_{m-1}, g y_{m}\right) \\
&+d\left(g z_{n}, g z_{n+1}\right)+d\left(g z_{n+1}, g z_{n+2}\right)+\cdots+d\left(g z_{m-1}, g z_{m}\right) \\
& \leq \frac{1}{\sqrt{a}} k^{n-n_{0}} f\left(g x_{n_{0}}, g y_{n_{0}}, g z_{n_{0}}\right)+\frac{1}{\sqrt{a}} k^{n+1-n_{0}} f\left(g x_{n_{0}}, g y_{n_{0}}, g z_{n_{0}}\right) \\
&+\cdots+\frac{1}{\sqrt{a}} k^{m-1-n_{0}} f\left(g x_{n_{0}}, g y_{n_{0}}, g z_{n_{0}}\right) \\
& \leq \frac{1}{\sqrt{a}} k^{n-n_{0}} \frac{1}{1-k} f\left(g x_{n_{0}}, g y_{n_{0}}, g z_{n_{0}}\right) \\
& \rightarrow 0, \text { as } n \rightarrow \infty .
\end{aligned}
$$

Hence $\left\{g x_{n}\right\},\left\{g y_{n}\right\}$ and $\left\{g z_{n}\right\}$ are Cauchy sequences in $(X, d)$. Suppose $g(X)$ is complete, there exist $u, v, w \in g(X)$ such that

$$
\lim _{n \rightarrow \infty} g x_{n}=u=g \alpha, \lim _{n \rightarrow \infty} g y_{n}=v=g \beta \text { and } \lim _{n \rightarrow \infty} g z_{n}=w=g \gamma
$$

for some $\alpha, \beta, \gamma \in X$.

Now, we show that $(g \alpha, g \beta, g \gamma)$ is triple coincidence point of $F$ and $g$.

Since $f$ is lower semi continuous from (2.8), we have

$$
\begin{aligned}
0 & \leq f(g \alpha, g \beta, g \gamma) \\
& =D(g \alpha, F(\alpha, \beta, \gamma))+D(g \beta, F(\beta, \alpha, \beta))+D(g \gamma, F(\gamma, \beta, \alpha)) \\
& \leq \lim _{n \rightarrow \infty} \inf f\left(g x_{n}, g y_{n}, g z_{n}\right)=0
\end{aligned}
$$

Hence, we get

$$
D(g \alpha, F(\alpha, \beta, \gamma))=D(g \beta, F(\beta, \alpha, \beta))=D(g \gamma, F(\gamma, \beta, \alpha))=0
$$

which implies that

$$
g \alpha \in F(\alpha, \beta, \gamma), g \beta \in F(\beta, \alpha, \beta) \text { and } g \gamma \in F(\gamma, \beta, \alpha)
$$

Thus $(\alpha, \beta, \gamma)$ is triple coincidence point of $F$ and $g$.

Example 2.4 Let $X=[0,1]$ and $d: X \times X \rightarrow \mathbb{R}$ by $d(x, y)=|x-y|$, then $(X, d)$ is complete metric space and we define $\preceq b y$

$$
x \preceq y \Longleftrightarrow x \leq y
$$

then $\preceq$ is partial order relation.

We define $g: X \rightarrow X$ by $g(x)=\frac{x}{2}, F: X \times X \times X \rightarrow C B(X)$ by $F(x, y, z)=[x, 1], \forall x, y, z \in X$.

Then

$$
\begin{aligned}
f(g x, g y, g z) & =D(g x, F(x, y, z))+D(g y, F(y, x, y))+D(g z, F(z, y, x)) \\
& =\inf \{d(g x, a): a \in[x, 1]\}+\inf \{d(g y, b): b \in[y, 1]\}+\inf \{d(g z, c): c \in[z, 1]\} \\
& =d\left(\frac{x}{2}, x\right)+d\left(\frac{y}{2}, y\right)+d\left(\frac{z}{2}, z\right) \\
& =\left|\frac{x}{2}-x\right|+\left|\frac{y}{2}-y\right|+\left|\frac{z}{2}-z\right| \\
& =\frac{x+y+z}{2} .
\end{aligned}
$$

Also let $\phi:[0, \infty) \rightarrow[a, 1], 0 \leq a<1$ by $\phi(t)=\frac{t}{t+1}$ it is clear that $\lim _{r \rightarrow t^{+}} \sup \phi(t)<1$ for each $t \in[0,+\infty)$.

Without loss generality we choose $g 0=g u \preceq g x, g 0=g v \preceq g y$ and $g 0^{+}=g w \preceq g y$.

It is clear that

$$
\sqrt{\phi(f(g x, g y, g z))}[d(g x, g u)+d(g y, g v)+d(g z, g w)] \leq f(g x, g y, g z)
$$

such that

$$
f(g u, g v, g w) \leq \phi(f(g x, g y, g z))[d(g x, g u)+d(g y, g v)+d(g z, g w)] .
$$

Hence all conditions of Theorem 2.3 are satisfied and $(0,0,0)$ is the coincidence point of $g$ and $F$. 
Corollary 2.5 Let $(X, d)$ be a metric space endowed with a partial order $\preceq$ and $\Delta \neq \emptyset$, that is there exist $\left(x_{0}, y_{0}, z_{0}\right) \in \Delta$. Suppose that $F: X \times X \times X \rightarrow C L(X)$ has a $\Delta$ - property such that $f: X \times X \times X \rightarrow[0,+\infty)$ given by

$$
f(x, y, z):=D(x, F(x, y, z))+D(y, F(y, x, y))+D(z, F(z, y, x)),
$$

is lower semi - continuous and that there exists a function $\phi:[0,+\infty) \rightarrow[a, 1), 0<a<1$, satisfying $\lim _{r \rightarrow t^{+}} \sup \phi(r)<$ 1 , for each $t \in[0,+\infty)$.

If for any $(x, y, z) \in \Delta$ there exist $u \in F(x, y, z), v \in F(y, x, y)$ and $w \in F(z, y, x)$ satisfying

$$
\sqrt{\phi(f(x, y, z))}[d(x, u)+d(y, v)+d(z, w)] \leq f(x, y, z)
$$

such that

$$
f(u, v, w) \leq \phi(f(x, y, z))[d(x, u)+d(y, v)+d(z, w)]
$$

Then, F has a triple fixed point.

Proof. If we take $g=I$ (identity map), then remaining proof follows from Theorem 2.3.

\section{Discussion and Conclusions}

The main Theorem 2.3 is an extension of Theorem of [6] from coupled coincidence point to tripled coincidence point. We have given an example to illustrate our main theorem. We also obtain a corollary from our Theorem 2.3 .

\section{REFERENCES}

[1] B.E. Rhoades, A fixed point theorem for a multi - valued non - self mapping, Comment. Math. Univ. Carolin., 37 (1996), $401-404$.

[2] B. Samet and C. Vetro, Coupled fixed point theorems for multi - valued nonlinear contraction mappings in partially ordered metric spaces, Nonlinear Analysis, 74 (2011), 4260 - 4268.

[3] H. Aydi, M. Abbas and M. Postolache, Coupled coincidence points for hybrid pair of mappings via mixed monotone property, J. Adv. Math. Stud., 5(1), (2012), No. 1, 118 - 126.

[4] I. Altun, A common fixed point theorem for multi - valued Círíc type mappings with new type compatibility, An. St. Univ. Ovidius Constanta., 17(2), (2009), 19 - 26.

[5] K.P.R.Rao, G.N.V.Kishore and K. Tas, A unique common triple fixed point theorem for hybrid pair of mappings, Abstract and Applied Analysis, Volume 2012, Article ID 750403, 9 pages, doi:10.1155/2012/750403.

[6] N. Hussain and A. Alotaibi, Coupled coincidences for multi - valued contractions in partially ordered metric spaces, Fixed Point Theory and Applications, 2011, 2011 : 82.

[7] N. Mizoguchi and W. Takahashi, Fixed point theorems for multi - valued mappings on complete metric spaces, J. Math. Anal. Appl., 141 (1989), 177 - 188.

[8] S.Banach, Surles operations dans les ensembles abstraits et leur applications aux equations, integrals, Fund. Math., 3(1922), 133 - 181.

[9] S. B. Jr. Nadler, Multi - valued contraction mappings, Pacific J. Math., XXX (1969), 475 - 488.

[10] V. Berinde and M. Borcut, Tripled fixed point theorems for contractive type mappings in partially ordered metric spaces, Nonlinear Analysis, 74(15), (2011), 4889 - 4897.

[11] Wei - Shih Du, Some generalizations of Mizoguchi - Takahashi's fixed point theorem, Int. J. Contemp. Math. Sci., 3 (2008) $1283-1288$. 